\title{
23. August - ein wichtiges Datum für die Sicherheit am Arbeitsplatz
}

\section{Michel Matter}

Dr. med., Vizepräsident der FMH, Departement Dienstleistungen und Berufsentwicklung

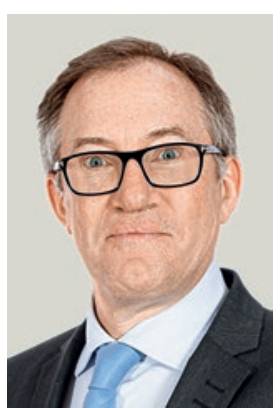

Seit mehreren Generationen führt die Familie Buckel in Genf einen Ocularisten-Betrieb - den letzten in der Westschweiz, der noch Augenprothesen aus Glas herstellt. Wie Matthias Buckel schildert, kam zu Beginn seiner beruflichen Laufbahn die Hälfte der Patientinnen und Patienten infolge eines Arbeitsunfalls zu ihnen. Mittlerweile ist dies sehr selten geworden, die Sicherheit am Arbeitsplatz ist viel höher als früher. Die Zeiten haben sich zum Besseren gewandelt.

In der Medizin wie auch in der Berufswelt hat Sicherheit am Arbeitsplatz nun oberste Priorität. Es gilt, alles $\mathrm{zu}$ tun, um potenzielle Risiken zu ermitteln und Gefahren zu beseitigen. Dies umfasst eine gewissenhafte Identifizierung von Risiken, ein zielgerichtetes Pflichtenheft der zu ergreifenden Massnahmen und vor allem eine auf dem neuesten Stand befindliche Dokumentation nach den staatlichen Arbeitsschutzvorschriften.

In Zusammenarbeit mit dem Schweizerischen Verein für Arbeitssicherheit und Gesundheitsschutz (Arbeitssicherheit Schweiz) hat die FMH eine Branchenlösung für Arztpraxen entwickelt, die individuell an jede Praxis und an jedes medizinische Zentrum angepasst werden kann. Dieses Ergebnis wäre ohne ein Gremium interdisziplinärer Expertinnen und Experten, die

In der Medizin wie auch in der Berufswelt hat Sicherheit am Arbeitsplatz nun oberste Priorität.

die Vielfalt unserer medizinischen und chirurgischen Fachgebiete widerspiegeln, nicht möglich gewesen. Ein grosses Dankeschön gebührt unseren Kolleginnen und Kollegen sowie Frau Sonia Barbosa, Expertin im Departement Dienstleistungen und Berufsentwicklung der FMH, für die investierte Zeit und ihre Überlegungen. Die Qualität des erzielten Ergebnisses in Form digitaler Module und seine Spezifität zeigen, dass das angestrebte Ziel, die Realität vor Ort abzubilden, erreicht wurde.

Am Anfang dieses gesetzlich gewollten Verfahrens stand der Bedarf von Kolleginnen und Kollegen, die sich in ihren Arztpraxen mit hohem Verwaltungsaufwand und unangekündigten kantonalen Kontrollen konfrontiert sahen; 2019 wurde von den kantonalen Ärztegesellschaften eine entsprechende Anfrage an uns gerichtet. Es gibt zwar eine Reihe von Branchenlösungen, bis jetzt aber noch keine speziell für Arztpraxen ausserhalb von Spitälern. Ärztinnen und Ärzte

\section{Es gilt, alles zu tun, um potenzielle Risiken} zu ermitteln und Gefahren zu beseitigen.

sind sowohl Gesundheitsfachleute als auch Arbeitgeber. Die juristische und medizinische Verantwortung, die sich daraus ergibt, liegt auf der Hand. Auch wenn dies für die Arztpraxen und medizinischen Zentren sicherlich mit entsprechenden Kosten einhergehen wird, ist die Einhaltung der Vorschriften der Eidgenössischen Koordinationskommission für Arbeitssicherheit rechtlich und administrativ unausweichlich. Die von FMH und Arbeitssicherheit Schweiz vorgeschlagene Branchenlösung ist dank dem erheblichen finanziellen Einsatz der FMH und dem Know-how der Expertinnen und Experten von Arbeitssicherheit Schweiz die kostengünstigste aller bekannten Lösungen im Gesundheitssektor und eindeutig diejenige, die unserer täglichen Realität am besten gerecht wird.

Die Sicherheit am Arbeitsplatz hat in unseren Praxen höchste Priorität. Die Branchenlösung der FMH sorgt dafür, dass Ärztinnen und Ärzte in ihrer Eigenschaft als Arbeitgeber ihrer gesetzlichen Verantwortung nachkommen und den Gesundheitsschutz ihres Personals und ihrer Patientinnen und Patienten in Praxen und medizinischen Zentren verbessern können. Dies ist ein wichtiger Schritt nach vorn. 